\title{
Semper Retis memorabile bellum (Raet. 7.565). Simon Lemnius in der Nachfolge des Silius Italicus
}

\author{
FLORIAN SCHAFFENRATH
}

\begin{abstract}
In his epic poem Raeteis, the poet Simon Lemnius (1522-1550) sings of the battles that took place in 1499 between the Raetians (Grisons) and the troops of Maximilian I and the Swabian League. The following article shows that Lemnius did not base his poetry primarily on Virgil's Aeneid, as often claimed in the past, but that Silius Italicus and his Punica are his main epic model. This is demonstrated by the following points: intertextual relations especially in the proem, the missing single hero, the populus as hero, historiographical source, comparisons of battles in the Raeteis with battles in the Punica.
\end{abstract}

Wenn der neulateinische Dichter Simon Lemnius (1511-1550)* heute noch bekannt ist, dann zumeist aufgrund des heftigen Disputes, den er noch als Student in Wittenberg mit keinem geringeren als Martin Luther austrug: Lemnius hatte zwei Bücher Epigrammata publiziert, ${ }^{1}$ gegen die Luther öffentlich so vehement vom Leder zog, dass sich Lemnius gezwungen sah, die Universität zu verlassen, und in der Folge eine Reihe tatsächlich höchst gehässiger Schriften gegen Luther verfasste. Nicht nur der heutige Leser schüttelt verwundert den Kopf, ${ }^{2}$ dass die im Grunde harmlosen Epigramme eine derartig heftige Reaktion auszulösen vermochten. Schon Joachim Camerarius d.Ä. (1500-1574) sah die Vorwürfe differenziert. ${ }^{3}$ Nicht nur rehabilitiert, sondern zudem noch nobilitiert wurde Simon Lemnius letztlich durch Gotthold Ephraim

* Eine frühere Version dieses Beitrages konnte ich im November 2021 bei einem Vortrag an der Universität Fribourg vorstellen. Ich danke David Amherdt für diese Gelegenheit. Außerdem bedanke ich mich bei allen, die sich rege an der Diskussion beteiligt haben, namentlich bei Didier Guex und Clemens Schlip, sowie bei den zwei anonymen Gutachtern, die mir wertvolle Anregungen geliefert haben.

${ }^{1} \mathrm{Zu}$ den Epigrammata des Lemnius vgl. L. Mundt, Lemnius und Luther. Studien und Texte zur Geschichte und Nachwirkung ihres Konflikts (1538/39) (Bern, 1983) und dazu ergänzend F. Wachinger, "Anmerkungen zu den Epigrammen des Simon Lemnius", Humanistica Lovaniensia 34B (1985), 114-132; W. Ludwig, "Zur Verbreitung und Bedeutung der Epigramme des Simon Lemnius", Daphnis 23 (1994), 659-664.

2 Ebenfalls der Meinung, dass Lemnius' Epigrammata harmlos seien, ist G. Vorberg (ed.), Simon Lemnius, Monachopornomachia. Der Mönchshurenkrieg. Threni, Klaggesang. Von der Sardoa (München, 1919), 5-11.

3 Vgl. Mundt 1983 (wie Anm. 1), Bd. 2, 342-343. 
Lessing (1729-1781), der den Bündner Autor als Musterbeispiel für einen Dichter, der sich durch sein Schaffen ins Unglück stürzte, hinstellt. ${ }^{4}$

Vor dem Hintergrund dieser Debatte fiel weit weniger Licht auf Lemnius' weitere Werke, von denen sich einige mehr Aufmerksamkeit der Forschung verdient hätten. Dies gilt insbesondere für sein dichterisches Hauptwerk, das Epos Raeteis, an dem Lemnius bis zu seinem Tod arbeitete und das im Zentrum der folgenden Ausführungen stehen wird. ${ }^{5}$ Die Grundthese wird folgende sein: An verschiedenen Stellen der Sekundärliteratur wird behauptet, dass sich Lemnius mit seiner Raeteis zu einem Bündner Vergil machen wollte. ${ }^{6}$ Dafür gibt es zwar Hinweise im Text, 7 doch ist dies nur begrenzt richtig, und zwar nur dann, wenn man den Namen "Vergil" hier für eine Metonymie, eine bloße Chiffre, für einen Epiker ganz allgemein nehmen möchte. In der Tat ist die Raeteis ein umfangreiches Epos in mehreren Büchern, das die kriegerischen Ereignisse des Jahres 1499 in Graubünden in die lange Tradition der epischen Dichtung einschreibt. Viel eher als Vergils Aeneis dienten Lemnius aber die Punica des Silius Italicus als Vorlage auf verschiedenen Ebenen. Verschiedene Aspekte dieser PunicaNachfolge herauszuarbeiten wird Aufgabe der folgenden Ausführungen sein.

4 Lessing würdigt das Schicksal des Lemnius in den ersten acht Briefen, die im Zweiten Teil der Schriften von 1753 erschienen sind: G. E. Lessing, Lessings Schriften. Zweyter Theil (Berlin, 1753), A2r-C5r bzw. [3]-75. Vgl. Mundt 1983 (wie Anm. 1), Bd. 1, xiii-xvii.

5 Die Raeteis ist immer noch zu benutzen in der Ausgabe von P. Plattner (ed.), Die Raeteis von Simon Lemnius. Schweizerisch-deutscher Krieg von 1499. Epos in IX Gesängen (Chur, 1874). Für diesen Aufsatz wurde zusätzlich für alle hier abgedruckten Zitate die Handschrift verglichen, die der Ausgabe von Plattner (dort Sigle T) zugrunde lag: Staatsarchiv Graubünden, Sig.: D V/3.210.001, ID: 479413. Zu den Schwierigkeiten, die eine neue kritische Ausgabe bisher verhindert haben, vgl. L. Mundt (ed.), Simon Lemnius, Bucolica. Fünf Eklogen (Tübingen, 1996), vii.

6 So schreibt etwa C. Willi, Calvenschlacht und Benedikt Fontana (Chur, 1971), 70 Lemnius habe mit seiner Raeteis "eine eigentliche rätische Aeneis" verfassen wollen.

7 Am Ende des Epos meint Lemnius in der Sphragis Andinum mihi patria rura dederunt (Raet. 9.1104) und setzt damit seine Heimat mit derjenigen Vergils, dem Dorf Andes bei Mantua, gleich. 


\section{Die Raeteis und ihr Autor}

Die Lebensgeschichte des Simon Lemnius (eigentlich Simon Lemm Margadant, latinisiert Emporicus) wurde wiederholt erzählt, meistens um ihn als einen der wenigen herausragenden humanistischen Autoren aus Graubünden $\mathrm{zu}$ würdigen. ${ }^{8}$ Seine Biographie, so interessant sie auch wäre, muss daher hier nicht erneut dargestellt werden. Sie spielt eine gewisse Rolle für das Verständnis der Raeteis, weil Lemnius mehrfach (wie bereits in seinen Amores $^{9}$ ) auf sich selbst und auf Details seines Lebens zu sprechen kommt.

An mehreren Stellen erwähnt er das Münstertal als seine Heimat, wo seine Familie einen Hof in Sta. Maria nahe dem Wasserfall Pischa bewirtschaftete; an der Hauswand des Hofes erinnert heute noch eine Gedenktafel an den berühmten Sohn. ${ }^{10}$ Lemnius erwähnt seine Eltern, die aufgrund

8 Zu Lemnius' Biographie vgl. Plattner 1874 (wie Anm. 5), iii-xix; Id., Raeteis. Heldengedicht in acht Gesängen von Simon Lemnius. Im Versmaß der Urschrift ins Deutsche übertragen (Chur, 1882), i-xvii; F. Vetter, "Simon Lemnius", Allgemeine Deutsche Biographie 18 (1883), 236-239; P. Merker, Simon Lemnius. Ein Humanistenleben (Straßburg, 1908); J. Michel, Die Quellen zur Raeteis des Simon Lemnius (Chur, 1914), 2-7; W. Ganß, "Die Raeteis des S. Lemnius (Ein Epos über den Schwabenkrieg)", Jahrbuch des Historischen Vereins für das Fürstentum Liechtenstein 55 (1955), 25-53, hier 26; R. Feller, E. Bonjour, Geschichtsschreibung der Schweiz. Vom Spätmittelalter zur Neuzeit (Basel, 1962), 147-149; P. Wiesmann, "Simon Lemnius 1511-1550", in Bedeutende Bündner aus fünf Jahrhunderten (Chur, 1970), Bd. 1, 109-126; Willi 1971 (wie Anm. 6), 69-70; Mundt 1983 (wie Anm. 1), Bd. 1, 3-9 und 38-45; L. Mundt (ed.), Simon Lemnius, Amorum libri IV (Bern, 1988), 8-14; L. Mundt, "Von Wittenberg nach Chur. Zu Leben und Werk des Simon Lemnius in den Jahren ab 1539", Daphnis 17 (1988a), 163-222; G. Deplazes, Die Rätoromanen. Ihre Identität in der Literatur (Disentis, 1991), 75-78; Mundt 1996 (wie Anm. 5), 1-6; V. Riedl, Antikerezeption in der deutschen Literatur vom Renaissance-Humanismus bis zur Gegenwart. Eine Einführung (Stuttgart, 2000), 62-63; J. Dittmar (ed.), Lyrik aus Liechtenstein. Von Heinrich von Frauenberg bis heute (Schaan, 2005), 377. Die vor 1874 erschienene biographische Literatur ist verzeichnet bei Plattner 1874 (wie Anm. 5), xxxiv.

9 Diesbezüglich relevant sind v.a. die autobiographischen Passagen amor. 3.2.207-226 und 4.1.53-66 sowie 4.2 (s.u.).

10 Raet. 1.128-136 (Praeterea est regio Raetei nominis arcta [T altra $\mathrm{T}^{1}$ arcta], / qua Ramus fluit et resonat rorantibus undis / rupe cadens gelido dispersus Pisa [T Pysa] liquore / hic quoque virginibus templum Vestalibus ingens / et sedes largae (pudor est retulisse nefandum / heu facinus), sacros temerandi milite campos / est animus; bellum mandataque nuntius affert, / at contra templum subitis Oeneia caterva / rebus defendunt, et sedes Marte tuentur), 1.345-346 (Iamque phalanx hastis, armisque potentibus intrat / Piseas valles et sternit milite campos) und 3.119-120 (Piseam vallem renovato Marte Tiroles [T Tyroles] / Invadunt). Auch der am Ende des Epos auftretende Sänger Musaeus, in dem sich Lemnius selbst darstellt, wird als Sohn des Münstertales bezeichnet (Raet. 9.971 Quem dea [T Dea] nutrivit Pisae rorantibus antris).

Humanistica Lovaniensia 70.2 (2021), 185-208 
der Kämpfe 1499 fliehen mussten, ${ }^{11}$ und im Besonderen seinen Vater, der mitgekämpft hat: ${ }^{12}$ In der Calven-Schlacht ist Vater Lemnius sogar der einzige, dessen Schild neben dem des Benedikt Fontana beschrieben wird. ${ }^{13}$ Neben ihm erwähnt Lemnius noch andere Familienmitglieder: seinen Onkel Nut Jenal (Nutius), einen Schmied und Meisterschützen, ${ }^{14}$ und andere Verwandte mütterlicherseits. ${ }^{15}$ Die häufigsten autoreferentiellen Verweise beziehen sich jedoch auf Lemnius den Dichter: Er steht in einer besonderen Beziehung zu den Musen, ${ }^{16}$ und er erscheint auf dem Schild, der im fünften Buch der Raeteis beschrieben wird; dort ist ein längerer Abschnitt (Raet. 5.359-399) Bischof Lucius Iter von Chur (reg. 1542-1549) gewidmet, an dem neben anderen Leistungen besonders hervorgehoben wird, dass er Lemnius in seiner Dichtung gefördert habe (Raet. 5.390 Ingeniis fautor, nostrae quoque gloria Musae). Lemnius lässt im neunten Buch den Sänger Musaeus auftreten und macht durch einige Angaben klar, dass hinter dieser Figur der Dichter gesehen werden soll. ${ }^{17}$ Schließlich ist noch die Sphragis des Epos $\mathrm{zu}$ nennen (Raet. 9.1093-1106): Dort streicht Lemnius seine dichterische Leistung für seine Heimat heraus und wünscht sich ewigen Ruhm für sein Gedicht.

Thema der Raeteis sind die kriegerischen Auseinandersetzungen, die sich 1499 in Graubünden zwischen Maximilian I. und den für ihn kämpfenden Truppen des Schwäbischen Bundes und der Tiroler auf der einen und den Bündnern (Raetern), mit denen die Eidgenossen verbündet

11 Raet. 4.1003-1008 (Ingressique meam patriam, vallemque [T Vallemque] Venustam / omniaque incendunt, flammis superaverat ignis. [...] Aufugere homines, et vos o cara [T ô chara] parentum / pectora; nec tum natus eram)

12 Raet. 7.623-624 (Lemnius hic unâ, cui fervida dextera bello, / qui pugnans olim devicit Marte Gigantem).

13 Raet. 6.265-281. Vulcanus, der Schöpfer dieses Schildes, hat darauf den Sohn des Lemnius, also den Dichter Simon Lemnius selbst, abgebildet, der dafür Lob und Ruhm für alle Zeiten erlangen werde, dass er die Kämpfe der Raeter besingen werde. In dieser Schildbeschreibung - der zweiten neben der Beschreibung des Schildes des Benedikt Fontana, die das gesamte fünfte Buch füllt - imaginiert Lemnius die Wirkung und Rezeption seines Gedichtes.

14 Raet. 6.167-180 und 7.227-252.

15 Raet. 7.630-634 (Tum Mercatantes [scil. Margadant] currebant rure bipenni, / maternumque genus, domus est Cocetia [scil. Conzett], et unâ [T unà] / fertur Janalis [scil. Jenal], patri socia arma ferebat, / pro patriaque prius pugnabant [T pugnarunt] fortiter armis, / virtutisque decus, patriae et meruere salutem.)

16 Raet. 7.316-318 und 337-339.

17 Raet. 9.967-1014. Schon Plattner 1874 (wie Anm. 5), 172 Anm. 1 erklärt: "Unter diesem Namen führt sich der Dichter selbst ein." 
waren, auf der anderen Seite abgespielt haben. ${ }^{18}$ Diese Kämpfe sind unter den Begriffen Calvenkrieg (benannt nach der entscheidenden Schlacht auf der Calven), Schwabenkrieg oder Schweizer Krieg in die Literatur eingegangen. ${ }^{19}$ Eine berühmte humanistische Schrift, Willibald Pirckheimers De bello Suitense sive Eluetico, ist einem Ereignis aus der Spätphase dieses Krieges gewidmet. ${ }^{20}$ Lemnius war kein Augenzeuge dieser Kämpfe, konnte sich aber auf Erfahrungen seiner Familie - sein Vater und andere Familienmitglieder waren, wie eben angesprochen, in die Kämpfe verwickelt - und auf historische Aufzeichnungen stützen, auf die noch genauer einzugehen ist.

Auch wenn Plattner die Raeteis in seiner Ausgabe bereits zusammengefasst hat, ${ }^{21}$ bietet es sich doch an, den Inhalt des Epos hier nochmals im Überblick zu bieten. Denn Plattners Zusammenfassung ist in Stichworten gehalten und fordert vom Leser keine geringen Vorkenntnisse. Außerdem lässt Plattner einige Elemente aus, auffälligerweise solche, in denen die Raeter in relativ schlechtem Licht erscheinen würden. ${ }^{22}$

Nach dem Proömium (1-12), in dem die Abwehrkämpfe der Raeter als Thema angegeben werden, beginnt das erste Buch mit einer Szene am Olymp (13-224): Juno bittet Juppiter um Hilfe für die Tiroler, die von den Raetern bedroht würden, Venus entgegnet, dass vielmehr die Raeter unter den Angriffen des Schwäbischen Bundes und der Tiroler zu leiden hätten, und stellt die Gründe für die jüngste Feindschaft zwischen Raetern und Tirolern dar. Die Erzählung wechselt vom Olymp nach Chur, wo die Bünde beschließen, Truppen gegen Maximilian aufzubieten (225-328). Die Raeter marschieren ins Münstertal und zwingen Bischof Heinrich von

$18 \mathrm{Zu}$ verschiedenen Jubiläen der Calven-Schlacht wurden in den letzten Jahrzehnten wiederholt Sammelbände vorgelegt: W. Lietha (ed.), Freiheit einst und heute. Gedenkschrift zum Calvengeschehen 1499-1999 (Chur, 1999); J. Riedmann (ed.), Calven 1499-1999. Bündnerisch-Tirolische Nachbarschaft (Bozen, 2001). Diese aktualisieren die älteren Darstellungen des Kriegsgeschehens, z.B. A. Jäger, Der Engedeiner Krieg im Jahre 1499. Mit Urkunden (Innsbruck, 1838).

19 Allgemein zum geschichtlichen Hintergrund: F. Pieth, Bündnergeschichte (Chur, $\left.1982^{2}\right), 94-103$.

20 Der Text wurde in einer deutschen Übersetzung von Ernst Münch herausgegeben von W. Schiel (ed.), Willibald Pirckheimer, Der Schweizerkrieg. Mit einer historischbiographischen Studie (Berlin, 1988). Eine Edition mit deutscher Übersetzung legte dann F. Wille (ed.), Willibald Pirckheimer, Historia belli Suitensis sive Helvetica (Baden, 1998) vor.

21 Plattner 1874 (wie Anm. 5), xxxv-xxxviii.

22 Ein Beispiel für eine solche Auslassung sind die Plünderungen in Schlanders (Raet. 8.257-283), die die Raeter nach ihrem Sieg dort durchführen, obwohl die Bevölkerung Lösegeld gezahlt hatte, um nicht geplündert zu werden.

Humanistica Lovaniensia 70.2 (2021), 185-208 
Chur, sie zu begleiten; vergeblich versucht er dort, sich abzusetzen (328-410). Die Raeter greifen das königliche Bergwerk in Scharlthal an und zwingen die Tiroler zur Zahlung von Lösegeld und zu einem Friedensschluss (411-485). Die Raeter besetzen die Fürstenburg, die Tiroler legen eine Besatzung nach Glurns. Urner kommen den Raetern für kurze Zeit zur Hilfe (486-543). Rudolf von Marmels erzählt in einer langen Rede (544-687) die Geschichte der Raeter von der Einwanderung der Etrusker in den Alpenraum bis hin zur Ausdehnung ihres Machtbereiches.

Das zweite Buch beginnt mit einer diachronen Beschreibung der Grenzen Raetiens (1-26). Juno stachelt in der Unterwelt, wo sie einige Helden, darunter auch den Stammvater der Raeter, sieht, die Furien auf, damit diese einen Krieg bei den Raetern ausbrechen lassen (27-61). Die Tiroler beginnen den Kampf von Feldkirch aus und nehmen die Luziensteig und Maienfeld ein. Die Eidgenossen kommen den Raetern zur Hilfe (62-175): Während sich am Olymp göttliche Hilfe für die Raeter abzeichnet, bringen sich auch die Eidgenossen in Stellung (176-211). Den Raetern und Eidgenossen gelingt die Rückeroberung der Luziensteig und von Balzers (212-383). Die Eidgenossen ziehen plündernd durch das Gebiet von Vaduz, wo Brandis gefangen genommen wird. Die Raeter können Maienfeld zurückerobern (384-541).

Im dritten Buch kommen die Ereignisse um Maienfeld zu ihrem Ende: Die Stadt wird geplündert, die Verantwortlichen für die kampflose Übergabe an die Tiroler bestraft (1-61). Die Raeter erobern weitere Orte und gewinnen neue Verbündete (62-118). Im Vinschgau erobern die Tiroler das Kloster Müstair. Der Bischof von Chur übergibt die Fürstenburg den Tirolern und setzt sich ab (119-232). Die Eidgenossen plündern im Walgau und können ein Heer des Schwäbischen Bundes bei Hard am Bodensee schlagen (233-294). Es kommt zu verschiedenen Streifzügen der Eidgenossen im Hegau und der Raeter in Naudersberg (295-378), aber auch der Truppen des Schwäbischen Bundes am Rhein (379-485). Die Tiroler haben bei Frastanz eine große Schanze errichtet, die Raeter und Eidgenossen zusammen angreifen. Die Anführer beider Seiten fallen (486-623).

Im vierten Buch setzt sich die Schlacht von Frastanz fort: Den Eidgenossen gelingt die Einnahme der Schanze, die Raeter machen diejenigen nieder, die nach Feldkirch fliehen wollen. Schließlich wüten die Eidgenossen im ganzen Walgau (1-131). Der Schwäbische Bund schickt neue Truppen nach Frastanz, doch die Eidgenossen bleiben siegreich (132-200). Die Tiroler greifen einige Orte im Vinschgau an und werden erst im Engadin von den Raetern zurückgetrieben (201-368). Es kommt zu heftigen Kämpfen zwischen Eidgenossen und dem Schwäbischen 
Bund am Rhein (369-684). König Maximilian greift nun selbst in die Kämpfe gegen die Eidgenossen ein (685-739). Es kommt zu weiteren Kämpfen in Dornach, Buffalora und Fuldera (740-908). Maximilian zieht auf der Calven starke Truppen zusammen (Katalog), und auch die Raeter bereiten sich dort auf eine große Schlacht vor (909-1078).

Das gesamte fünfte Buch ist eine Ekphrasis des Schildes, um den Venus ihren Gatten Vulcanus für den raetischen Helden Benedikt Fontana bittet. Auf dem Schild ist die Geschichte der Raeter und die Geschichte der Bischöfe von Chur zu sehen. Der zur Zeit der Calven-Schlacht regierende Bischof Heinrich V. von Hewen wird genannt und der Sieg der Raeter in der Schlacht vorhergesagt. Außerdem werden noch zwei Bischöfe genannt, die aus der Erzählperspektive erst in der Zukunft wirken sollten, Paul Ziegler und Lucius Iter, die den Dichter Lemnius unterstützten bzw. unterstützen sollten. Am Ende des Buches nimmt Fontana den Schild, kann aber das auf ihm Abgebildete - wie sein Vorbild Aeneas nicht verstehen.

Nachdem am Ende des vierten Buches ein Katalog der Truppen Maximilians geboten wurde, werden am Beginn des sechsten Buches nach einem Musenanruf die im Münstertal versammelten Truppen der Raeter mit ihren hervorragenden Kämpfern geschildert (1-180). Im raetischen Kriegsrat schlägt Paul von Capol einen Zangenangriff gegen die Schanze auf der Calven vor (181-238). So schlägt sich ein Teil der Raeter über das Gebirge und nimmt Schleis und Laatsch ein (239-480). Währenddessen greift die Hauptmacht der Raeter den Wall auf der Calven an; unter verschiedenen Aristien ragt diejenigen heraus, in der Benedikt Fontana fällt (481-699).

Auch zu Beginn des siebten Buches wird weiterhin in zahlreichen Einzelbildern gezeigt, wie die Hauptmacht der Raeter gegen den Wall stürmt (1-310). Nach einem Musenanruf wird geschildert, wie den Raetern schließlich die Erstürmung des Walles gelingt (311-510). Die Kämpfe enden und die Raeter können einigen Flüchtigen nachsetzen (511-642). Die Sieger feiern und ziehen plündernd nach Hause $(643-845)$.

Im achten Buch feiern die Raeter ihren Sieg in Chur, während vor Ort die Gefallenen bestattet werden (1-81). Erneut stellt Maximilian Truppen auf, die von verbündeten Eidgenossen und Raetern geschlagen werden können (82-292). Es folgen weitere kleinere Kämpfe bei der Luziensteig, im Hegau und am Rhein (293-453). Es kommt zur Schlacht bei Dornach, in der die Eidgenossen die Königlichen in die Flucht schlagen (454-604).

Im neunten Buch flammen die Kämpfe um Dornach wieder auf, und wieder bleiben die Eidgenossen siegreich (1-295). Bei der Siegesfeier 
besingt ein Sänger die Schweizer Geschichte von Rudolf von Habsburg bis zu den Kämpfen gegen den Schwäbischen Bund (296-568). Friedensverhandlungen in Basel werden immer wieder von kleineren Kämpfen unterbrochen (569-702). Schließlich kommt es auf göttliche Vermittlung zum Friedensschluss (703-726). Junge Engadiner besingen den überstandenen Krieg (727-924). In Chur werden Spiele veranstaltet; neben verschiedenen sportlichen Disziplinen tritt auch ein Sänger auf (925-1092). Das Gedicht endet mit einer Sphragis, in der der Dichter den geschilderten Ereignissen ewigen Ruhm verkündet (1093-1106).

Die Makrostruktur des Epos ist in Ringen um das zentrale fünfte Buch konstruiert: Die Randbücher 1 und 9 sind klar als Beginn und Ende markiert: Im ersten Buch hält unmittelbar nach dem Proömium Juno einen inneren Monolog, in dem sie die Hintergründe und Voraussetzungen für das folgende Geschehen erläutert; seit Vergils Aeneis und Silius Italicus' Punica beginnen viele Epen mit Aktionen der Juno, die als Gegnerin des eigentlichen Helden die Handlung in Gang setzt. ${ }^{23}$ Am anderen Ende des Gedichtes positioniert Lemnius eine Sphragis und die epischen Spiele, mit denen die Bündner den erfolgreichen Abschluss der Kämpfe feiern (Raet. 9.925-1092). Bereits in Homers Ilias markieren die Spiele das Ende (Il. 23: Wettkämpfe für Patroklos) und zahlreiche neuere Epiker sind Homer darin gefolgt und schildern ihre Spiele, anders als Vergil in der Mitte seines Epos (Aen. 5), am Ende des Werkes. ${ }^{24}$ Gleich wie Anfang und Ende markiert Lemnius auch die Mitte seines Werkes durch ein besonderes Strukturelement: Das gesamte fünfte Buch enthält keine eigentliche Handlung, sondern besteht aus einer gewaltigen Ekphrasis, in der der Schild beschrieben wird, den Vulkan für Benedikt

23 Aen. 1.34-49 und Pun. 1.21-139. Hier nur wenige Beispiele für dieses Motiv in neulateinischen Epen, deren Abfassung der Raeteis vorangeht: In Antonio Baratellas Polydoreis drängt Juno am Beginn des Epos in der Unterwelt auf die Vernichtung Trojas (Pol. 1.1-72). In der 1521 in Rimini gedruckten Venetias des Publio Francesco Modesti blickt am Beginn Juno mit Argwohn auf das neu gegründete Venedig und schwört, diese Stadt zu vernichten (Ven. 1.71-120). In Francesco Rococciolos Alphonsias (1527/28) erscheint Juno dem Alfonso zu Beginn des Gedichtes im Schlaf und tadelt ihn wegen seiner Untätigkeit (Alf. 33-89).

24 Ein Beispiel ist hier Riccardo Bartolinis Austrias (Straßburg, 1516), in der im zwölften und letzten Buch ausführlich (Austr. 12.173-955) Spiele als Abschluss des Landshuter Erbfolgekrieges geschildert werden. Allgemein zu Spielen im Epos vgl. H. Lovatt, "Epic Games. Structure and Competition”, in C. Reitz, S. Finkmann (ed.), Structures of Epic Poetry, Bd. 2.1: Configuration (Berlin - Boston, 2019), 409-445. 
Fontana fertigt. ${ }^{25}$ Rund um dieses fünfte Buch, d.h. am Ende des vierten Buches (Raet. 4.1013-1078) und am Beginn des sechsten Buches (Raet. 6.4-75), finden sich zwei große Truppenkataloge, in denen die Kämpfer des Gotteshausbundes (4. Buch) und des Oberen und des Zehngerichtenbundes (6. Buch) aufgezählt werden. Die Bücher vier und sechs sind zudem dadurch verbunden, dass in ihnen die zentrale Schlacht des Krieges von 1499, die Schlacht auf der Calven, geschildert wird. Ein letztes auffälliges makrostrukturelles Merkmal der Raeteis ist es, dass die Buchgrenzen häufig dadurch verwischt werden, dass eine Schlacht am Ende des einen und am Beginn des nächsten Buches geschildert wird (2/3: Maienfeld, 3/4: Frastanz; 8/9: Dornach).

Lemnius starb, ehe er die Arbeit an der Raeteis abschließen konnte. Dies mag einige Ungereimtheiten in der Darstellung und einige formale Probleme erklären. So ist etwa keine der insgesamt 82 Reden des Epos umfangreich ausgearbeitet. Auch in den Gleichnissen hätte Lemnius wohl noch die Feile angelegt und wahrscheinlich einige der insgesamt fünf (!) Kapaneus-Gleichnisse gestrichen, von denen sich allein drei auf engstem Raum im siebten Buch finden. ${ }^{26}$ Besonders verstörend für den Leser ist die Rolle, die Fontana während der Schildbeschreibung im fünften Buch spielt: ${ }^{27}$ Am Beginn des Buches wird nur geschildert, dass Venus sich auf die Liparischen Inseln begibt, um ihren Gatten um Waffen für Fontana zu fragen, die Vulcanus nach einer feurigen Liebesnacht auch anfertigt (Raet. 5.1-8). Doch dann heißt es am Ende des Buches (Raet.

25 Dabei handelt es sich allerdings um einen Eingriff Plattners in den Text. In der Handschrift $\mathrm{T}$ endet das fünfte Buch nicht mit dem staunenden Fontana, sondern es schließen sich der Musenanruf (Raet. 6.1-10) und der Beginn des Kataloges an, die in der Ausgabe von Plattner den Beginn des sechsten Buches markieren. Folgende Verse, die in der Handschrift am Ende des 5. Buches nach dem Musenanruf folgen, fehlen in Plattners Ausgage ganz: Illos campus alit, lustra sitimque [sic!] condunt cana / avertunt fluvio, manibus adducitur unda / [f. 87v] quos Fontes Thuscisque Altrix Hetrusca Rhetorum / et Rhetium sedes, arx hinc quoque Retia celsa / dicta quibus monitor iuvenis de marmore natus / Hannus erat crescens teneris tum floribus annis / non illis solitum Pharetram implevisse sagittis / at longis portare habiles mucronibus enses / quique nemus densum bifidi quique ostia Rheni / fontis amant tepidique sedent in valle recurva. / Horrebant telis Hilandia moenia parva / dicta aliter Rheto. Nam quondam dixit Iantem. / Additur his Foveae plebes Saxique cohortes / Saxi quod supra est, linguâ Germana propago. / Nec non est sylvae Castrum Andesteque Rubisque / vos etiam Slanzae, vos septima, Luveneque.

26 Der Mythos von Kapaneus vor Theben ist der Inhalt folgender Gleichnisse: Raet. 4.168-169; 6.537-539; 7.65-66; 7.329-332; 7.407-408.

27 Michel 1914 (wie Anm. 8), 148 bemängelt außerdem, dass von den Waffen, die im 5. Buch für Fontana erst geschmiedet werden, bereits früher (Raet. 1.619) die Rede war. Allerdings wird an der genannten Stelle im 1. Buch nur ganz allgemein von arma gesprochen, sodass es sich nicht unbedingt um die von Vulkan gefertigten Waffen handeln muss.

Humanistica Lovaniensia 70.2 (2021), 185-208 
5.400-405), dass Fontana das auf dem Schild Dargestellte bewundert und sich dann - gleich wie Aeneas (Aen. 8.729-731) - die Waffen umhängt. War Fontana die ganze Zeit dabei?

\section{Die Erzählung vom Stammvater Raetus}

Überblickt man die Entwicklung der Gattung des neulateinischen Epos im 16. Jahrhundert, ${ }^{28}$ so erkennt man, dass sich in dieser Zeit die meisten Autoren in ihren Werken stark an Vergil und seines Aeneis orientiert haben. Auch Lemnius hätte sich in seiner Darstellung an der Aeneis ausrichten und die Urgeschichte der Raeter erzählen können, von der aus sich über verschiedene Techniken Bezüge zur späteren Geschichte herstellen ließen. Viele andere Epiker des 16. Jahrhunderts haben es so gemacht, Pierre de Ronsard (1524-1585) ist mit seiner Franciade (1572) wohl der bekannteste unter ihnen: Das König Karl IX. von Frankreich gewidmete Epos erzählt die Geschichte von Francus, dem mythischen Gründer Frankreichs, dessen Wirken in vielfältiger Weise mit der historischen Wirklichkeit Ronsards verbunden wird. ${ }^{29}$

Lemnius setzt mehrfach zur Erzählung eines solchen Urmythos an, bot sich ihm doch in den historiographischen Quellen zur raetischen Geschichte ein entsprechender heros ktistes an: Aegidius Tschudi (1505-1572) erzählt am Beginn seines Werkes Die uralt warhafftig Alpisch Rhetia (Basel 1538) im Kapitel Wie die Rhetier, yetz Churwalhen genant, uss Italia kommen, und Thuscaner gewesen, ouch von Rheto irem Houptman, und etlichen Geschlechten, dass zur Zeit der Galliereinfälle in Italien die Thuscier (Etrusker) zum Auswandern gezwungen wurden. Angeführt von Rhetus zogen sie in die Alpen, wo sie sich nach anfängli-

28 Es liegen mehrere Überblicksdarstellungen zum neulateinischen Epos vor: H. Hofmann, "Von Africa über Bethlehem nach America. Das Epos in der neulateinischen Literatur", in J. Rüpke (ed.), Von Göttern und Menschen erzählen. Formkonstanzen und Funktionswandel vormoderner Epik (Stuttgart, 2001), 130-182; C. Kallendorf, "The NeoLatin Epic", in Ph. Ford, J. Bloemendal, C. Fantazzi (ed.), Brill's Encyclopaedia of the Neo-Latin World, Bd. 1 (Leiden - Boston, 2014), 449-460; F. Schaffenrath, "Narrative Poetry", in S. Knight, S. Tilg (ed.), The Oxford Handbook of Neo-Latin (Oxford, 2015), 57-72; P. Gwynne, "Epic", in V. Moul (ed.), A Guide to Neo-Latin Literature (Cambridge, 2017), 200-220.

29 Für eine allgemeine Darstellung der Franciade Ronsards vgl. E. Rohmer, Das epische Projekt. Poetik und Funktion des "carmen heroicum" in der deutschen Literatur des 17. Jahrhunderts (Heidelberg, 1998), 134-164 mit einer Inhaltsangabe der vier erschienenen Bücher ebendort 139-141. 
chen Kämpfen niederließen und fortan nach ihrem Anführer "Rhetier" nannten.

Lemnius, der mit dieser Geschichte vertraut war, ${ }^{30}$ erkannte ihr Potential, ihren Helden Rhetus in einer epischen Dichtung in einen zweiten Aeneas zu verwandeln. Die Art und Weise, wie er die Geschichte der Auswanderung der Thuscier erzählt, erinnert in Vielem an die Auswanderung der Aeneaden aus Troja (1.581-586):

Ut fuerat Tuscis armis interrita pubes, attamen his acie Ticini ad fluminis undas fusi cesserunt Raeto duce Martis Hetrusci. Qui tum Maeoniis profugus discessit ab oris, Tyrrhenique viri, genus unde, et Raetica rura, Raeteique patres canaeque exordia gentis.

583 Raeto: $\mathbf{T}$ Rheto : $\mathbf{T}^{\mathbf{1}}$ Raeto | $\mathbf{5 8 5}$ Raetica: $\mathbf{T}$ Rhetica | $\mathbf{5 8 6}$ Raeteique: T Rheteique canaeque $\mathbf{T}^{\mathbf{1}}$ Canaeque

Obwohl die Tuscier eine Jungmannschaft hatten, die sich im Kampf nicht schrecken ließ, mussten sie diesen [sc. den Galliern] in der Schlacht an den Wellen des Flusses Ticino doch weichen. Unter ihrem Anführer im Krieg Raetus zogen die Etrusker ab. Er flüchtete damals und zog sich aus den mäonischen Landen zurück, zusammen mit den tyrrhenischen Männern, woher das Geschlecht und das raetische Land kommt, die raetischen Väter und der Ursprung dieses uralten Stammes.

Nicht nur das Wortmaterial dieser Passage ist dem Proömium der Aeneis entnommen (vgl. Aen. 1.1 ab oris; 1.2 profugus; 1.6 genus unde; 1.7 Albanique patres), sondern auch die Gedankenführung verläuft parallel: Nach heftigen Kämpfen muss ein Held die Flucht ergreifen; er nimmt neues Land in Besitz und wird dort zum Stammvater eines mächtigen Volkes. Doch anders als in der Aeneis wird die Raeteis nicht aus der Perspektive dieses Gründungsheros erzählt, von der aus sich geschichtliche Durchblicke in die Zukunft auftun, sondern es wird auf die Geschichte dieses Helden lediglich zurückgeblickt. In der Fortsetzung der eben zitierten Stelle heißt es, dass sich "noch jetzt" (Raet. 1.587 nunc etiam) der Bezug zu Raetus in der Sprache der Raeter zeige und dass

30 Zu Tschudi als Quelle für Lemnius' Raeteis vgl. Michel 1914 (wie Anm. 8), 104-118, bes. 106 .

Humanistica Lovaniensia 70.2 (2021), 185-208 
zahlreiche aktuelle Familien auf ihre etruskischen Wurzeln stolz seien. Auch an den weiteren Stellen, an denen Lemnius schlaglichtartig auf die Geschichte von Raetus zu sprechen kommt, wird sie nur kurz als Referenzpunkt angerissen, mit dem sich eine aktuelle Familie oder ein Geschlecht in Verbindung bringen möchte (Raet. 1.35-38; 2.33; 2.50; $2.122-123 ; 3.218$; 4.1026-1027; 5.15-16; 5.163-164; 9.1099-1100).

Nur an zwei Stellen der Raeteis wird die Geschichte des Raetus nicht nur in einer kurzen Anspielung, sondern etwas ausführlicher erwähnt. Diese beiden Stellen werden nicht vom allwissenden Erzähler des Epos, sondern von einem intradiegetischen Erzähler vorgetragen: Sie sind in den ersten historischen Rückblick der Juno am Olymp (Raet. 1.13-53) und in die Rede des Rudolf von Marmels (Raet. 1.544-687) eingebaut. Lemnius lässt sich also Figuren, die für kurze Zeit die Rolle des Erzählers bzw. Dichters übernehmen, an einem vergilischen Konzept versuchen, während er selbst einen anderen Weg geht.

\section{Silius als Vorbild}

In seiner 1914 erschienenen Monographie zur Raeteis hat Janett Michel klar zeigen können, dass Silius Italicus (neben Homer, Vergil und Statius) ein wichtiges antikes Vorbild für Lemnius war. ${ }^{31}$ Wie in Arbeiten aus dieser Zeit üblich, erbringt Michel diesen Nachweis, indem er eine Reihe von textlichen Parallelen in den beiden Epen einander in wörtlichen Zitaten gegenüberstellt. Diese mühsame und verdienstvolle Arbeit muss also hier nicht wiederholt werden. Neben solchen intertextuellen Bezügen lassen sich aber noch mehr Bezüge zwischen den Punica und der Raeteis herstellen und für eine Gesamtinterpretation des Werkes fruchtbar machen, was im folgenden Abschnitt versucht wird.

\section{Proömium}

Dass Lemnius literarisch in andere Fußstapfen als in die Vergils treten möchte, wird schon in den ersten Zeilen der Raeteis offensichtlich. Das Proömium des Epos lehnt sich stark an das der Punica des Silius Italicus an, worauf grundsätzlich schon Janett Michel hingewiesen hat (Raet. 1.1-12): ${ }^{32}$

31 Vgl. Michel 1914 (wie Anm. 8), 17-52.

32 Michel 1914 (wie Anm. 8), 17-18 druckt hintereinander das Proömium der Raeteis und der Punica ab, gibt aber keine weiteren Erklärungen zu den Verbindungen zwischen diesen beiden Texten. 
Fortia Raeteis cantata nepotibus arma ordior, unde virûm se tollit gloria terris. Da mihi, Musa, decus, da tantae proelia cladis et memorare Athesis rorantes sanguine rivos, ac tria Raetorum sectas in foedera gentes, quot bellatores, quantosque in bella crearint, aëriasque inter quae gesserit arduus Alpes Raeticus Hetrusca deductus origine miles, utque acies clausas vallo perfregerit alto, dum quondam patrios defendit Raetia campos. Fas aperire mihi et servata minoribus arma tradere et ingentis causas narrare tumultus.

$\mathbf{2}$ ordior: $\mathbf{T}$ ordiar $\mid \mathbf{9}$ acies: $\mathbf{T}$ aties : $\mathbf{T}^{\mathbf{1}}$ acies

Ich beginne mit den tapferen Kämpfen, besungen für die Nachkommen der Raeter, von wo sich die Ehre dieser Männer über alle Länder verbreitete. Verleihe mir, oh Muse, Anmut, lass mich an die Schlachten dieses großen Krieges erinnern, an die vor Blut triefenden Fluten der Etsch, an die drei Bünde der in verschiedene Stämme geteilten Raeter, wie viele und wie bedeutende Kämpfer sie für die Kriege hervorgebracht haben, die das Heer der Raeter, die sich von den Etruskern herleiten, hoch in den luftigen Alpen geführt hat! Lass mich daran erinnern, wie sie in einem tiefen Tal eine bewaffnete Schanze durchbrochen haben, als Raetien einst seine heimischen Fluren verteidigte! Lass mich all das verkünden, die für die Nachkommen bewahrten Kämpfe überliefern und die Gründe dieses großen Krieges erklären!

Es ist nicht nur das (unterstrichen markierte) Wortmaterial, das hier aus dem Proömium der Punica wieder auftaucht und neu zusammengesetzt wird (vgl. Pun. 1.1 ordior; arma; se gloria tollit; 1.3 da, Musa, decus; memorare; 1.4 quantosque ad bella crearit; 1.5 quot viros; 1.6 gens; 1.9 foedus; 1.13 gentes; 1.17 causas; 1.18 servatum; 1.19 fas aperire mihi). Weitere Elemente führen den Leser schnell zu Silius Italicus: Es ist eben nicht das aus der Aeneis entnommene Signalwort cano, das am Beginn so vieler neulateinischer Epen steht, sondern das in der klassischen lateinischen Epik für Silius allein markante ordior, mit dem er sein Schaffen anfänglich beschreibt. Anders als in der Aeneis, aber gleich wie in den Punica folgt der Musenanruf gleich im dritten Vers nach einer 
allgemeinen Themenangabe. ${ }^{33}$ Die erstaunlichste Anpassung an das Punica-Proömium besteht im Motiv der Dreiheit: Silius erwähnt, dass es in Summe drei Punische Kriege gegeben hat (Pun. 1.8 ter Marte sinistro [...]), Lemnius führt an, dass sich drei Bünde für den Krieg gegen die Königlichen zusammengeschlossen hätten, und meint damit den Gotteshausbund, den Zehngerichtenbund und den Oberen Bund. ${ }^{34}$ Schließlich enden beide Proömien mit einer Überleitung zu den causae, den Kriegsgründen, die es vor den eigentlichen Ereignissen zu schildern gelte. - Während Vergil bereits im zweiten Wort seines Epos seinen Helden Aeneas anspricht (Aen. 1.1 virumque), findet sich im Proömium der Punica kein derartiger Hinweis. Das führt zur nächsten Gemeinsamkeit zwischen Punica und Raeteis: Es gibt keinen individuellen Helden.

\section{Held}

Während es einfach ist, in Odysseus den Helden der Odyssee oder in Aeneas den Helden der Aeneis festzumachen, ist die Frage nach dem Helden der Punica nicht so einfach zu beantworten: ${ }^{35}$ Am häufigsten präsent ist freilich Hannibal, doch er verkörpert alles Negative, nicht die positiv konnotierten Werte, die Silius vermitteln möchte. So kam die Forschung auf die Idee, im römischen Volk (populus Romanus) einen kollektiven Helden zu sehen, der sich zwar in verschiedenen Heldenfiguren manifestiert, der aber als ein ganzes zu sehen sei.

Etwas Vergleichbares findet sich in der Raeteis: Sie besitzt keinen singulären Helden in der Art eines Odysseus oder Aeneas. Vielmehr ist es das Volk der Raeter selbst, das die Rolle des Helden einnimmt. Sie werden als Raeti oder (je nach metrischem Bedarf) als Raetei bezeichnet, ${ }^{36}$ seltener werden sie als gens Raetea, als populus Raetus ${ }^{37}$ oder in Anspielung auf ihre Herkunft als gens Tyrrhena, gens Hetrusca

33 Auch in Statius' Thebais werden die Musen bereits im dritten Vers genannt, jedoch in Form einer Frage, nicht wie bei Silius Italicus und bei Lemnius mit einer Aufforderung.

34 Vgl. R. Sablonier, "Graubünden um 1500. Grundlagen und Horizonte", in Riedmann 2001 (wie Anm. 18), 95-119, hier 95.

35 Generell zur Frage des Helden der Punica vgl. R. Marks, From Republic to Empire. Scipio Africanus in the Punica of Silius Italicus (Frankfurt a.M., 2005), 61-67.

36 Als Volk werden die Raet(e)i kollektiv genannt: Raet. $1.59 ; 74 ; 85 ; 101 ; 117 ; 138$; $152 ; 174 ; 205 ; 209 ; 229 ; 466 ; 491 ; 539 ; 550 ; 636$ usw. (weitere 226 Nennungen in den folgenden Büchern).

37 gens Raetea: Raet. 1.14; 118; 280; 3.11-12; 80; 4.1017; 6.430; 8,294 bzw. populus Raetus: Raet. $1.142 ; 5.12 ; 7.717$. 
oder gens Lydia ${ }^{38}$ bezeichnet, einmal als gens nostra (Raet. 9.74). Einmal heißen sie sogar (in Anspielung auf den vir der Aeneis) Raetei viri (Raet. 9.740). Die heldenhaften Taten und Leistungen werden also nicht von einem stellvertretenden Mann vollbracht, sondern von einem Kollektiv, vom gesamten Volk der Bündner.

Das soll nicht heißen, dass in der Raeteis keine einzelnen heldenhaft agierenden Figuren auftreten: Als Repräsentanten des rätischen Volkes in seiner Gesamtheit treten einzelne Familien in Erscheinung, etwa die Planta (z.B. Raet. 1.39; 602; 647; 656), die Marmels (z.B. Raet. 1.161; 544) oder die Capol (z.B. Raet. 1.311). Doch keine einzige dieser Familien nimmt eine so zentrale und verbindende Rolle ein, dass man sie oder einen ihrer Vertreter als den zentralen Helden des Gedichtes ansehen könnte. Diese Rolle bleibt dem kollektiven Volk vorbehalten.

Dieser kollektive Zugriff auf die Akteure der Kriegsereignisse von 1499 beschränkt sich nicht nur auf die Raeter. Auch die mit den Raetern verbündeten Eidgenossen werden immer wieder in ihrer Gesamtheit als Helvetii adressiert, besonders auffällig in der eingelegten Schweizergeschichte (Raet. 9.296-568). Mutatis mutandis gilt dies auch für die Gegner der Raeter, die Tiroler oder die Mitglieder des Schwäbischen Bundes. Die Gestalt Maximilians I. ragt zwar als Einzelperson heraus und erhält eine (vor dem Hintergrund der Gesamtintention des Werkes nur schwer zu deutende) sehr positive Charakterisierung (Raet. 4.719-739), doch tritt Maximilian letztlich nur sporadisch in Erscheinung, ist also weit davon entfernt, der ein turnus- oder hannibalgleiche Gegner der Bündner zu sein.

Auch wenn er nicht der eine Held der Punica ist, so sticht Scipio doch unter den verschiedenen Figuren des Gedichtes heraus. Er hat kürzere Auftritte ab Buch 4 und spielt dann in den Büchern 15-17 des Epos eine so tragende Rolle, dass ihn Raymond Marks als "synecdochic hero"39 bezeichnen kann, der die Tugenden und Werte des römischen Volks in sich vereint und verkörpert.

Einen solchen Helden, der sich im Laufe der Darstellung immer mehr als Verkörperung des rätischen Volkes herauskristallisiert, gibt es in der Raeteis, wie ausgeführt, nicht. Dennoch trägt Benedikt Fontana, oder Fontaneus, wie er bei Lemnius heißt, einige Züge einer solchen Figur: Er tritt immer wieder sporadisch in Erscheinung (Raet. 4.836; 6.79), ehe er in der Schlacht auf der Calven die entscheidende Rolle spielt. Nur sein

38 gens Tyrrhena: Raet. 1.156-157; gens Hetrusca: Raet. 1.178; 5.24; gens Lydia: Raet. 1.173 .

39 Marks 2005 (wie Anm. 35), 82. 
beherzter Aufruf an seine Truppen, die Schanze der Königlichen furchtlos zu erstürmen, führt letztlich zum Erfolg, auch wenn Fontana selbst in diesen Kämpfen fällt.

\section{Historiographische Quelle}

Eine weitere Gemeinsamkeit zwischen den Punica und der Raeteis besteht darin, dass beide Epen stark von einer historiographischen Quelle abhängen. ${ }^{40}$ Es wurde - nach einer gewissen Unsicherheit der Forschung im 19. Jahrhundert - wiederholt festgestellt, dass das Geschichtswerk des Livius die Hauptquelle für die Punica ist. ${ }^{41}$ Freilich ist Silius Italicus weit davon entfernt, ein reiner Versifikator des Livius zu sein: Er versteht es gut, v.a. durch die Benutzung von Vergils Aeneis, ein Epos zu schaffen, kein in Verse gegossenes Geschichtswerk. Doch konnte auch gezeigt werden, dass Livius mehr als nur der Lieferant für historische Fakten und Details für Silius Italicus war.

Für Lemnius stellt folgende Schrift die wichtigste historiographische Quelle dar, der er teilweise bis ins Detail folg: ${ }^{42}$ Acta des Tyroler-Kriegs oder Argument der Krieg, Raub, Brandt, Angriff und Todtschlag, Anfangs zwüschendt der Hochwürdigen Stifft Chur an einem, und der Graffschaft Tyrol am andern, und weiter zwüschendt dem Römischen Khünig Maximilian, dem Schwäbischen großen Pundt, als Grafen und Herren zu Tyrol, an einem, ouch gemeinen Eidtgnoßen als gewandten der dreyen Pündt in Churwalchen, in dem 1499sten Jar geübt und beschenen. ${ }^{43}$ In diesem anonymen Kriegsbericht werden alle Ereignisse, die sich zwischen dem 10. Januar und dem 22. Juli 1499, also der Schlacht bei Dor-

40 Auf diese Gemeinsamkeit zwischen Silius Italicus und Lemnius hat bereits Michel 1914 (wie Anm. 8), 221 hingewiesen.

$41 \mathrm{Zu}$ Livius als Quelle für Silius Italicus vgl. J. Nicol, The Historical and Geographical Sources Used by Silius Italicus (Oxford, 1936); H.-G. Nesselrath, "Zu den Quellen des Silius Italicus", Hermes 114 (1986), 203-230.

42 In seinem Urteil etwas zu hart ist F. Vetter, "Die Quellen zur Geschichte der Schlacht an den Kalven", Anzeiger für schweizerische Geschichte 4 (1882-1885), 258-278, hier 277, wenn er von einem "sklavisch nachdichtende[n] Lemnius" spricht.

43 Allgemein zu den Acta vgl. Michel 1914 (wie Anm. 8), 84-104; Feller, Bonjour 1962 (wie Anm. 8), 144; A. Gutmann, Die Schwabenkriegschronik des Kaspar Frey und ihre Stellung in der eidgenössischen Historiographie des 16. Jahrhunderts (Stuttgart, 2010), 160-166. Die Schrift liegt in drei unterschiedlichen modernen Ausgaben vor: C. von Moor, C. Kind, "Acta des Tyroler-Kriegs", Rätia. Mittheilungen der Geschichtsforschenden Gesellschaft von Graubünden 4 (1869), 111-149; F. Vetter, "Benedikt Fontana. Eine schweizerische Heldenlegende", Jahrbuch für schweizerische Geschichte 8 (1883), 201-305, hier 276-300; C. Jecklin, Die Acta des Tirolerkriegs nach der ältesten Handschrift als Beitrag der Kantonsschule zur Calvenfeier (Chur, 1899). 
nach, zugetragen haben, aus bündnerischer Perspektive geschildert. Janett Michel hat es unternommen, in einer tabellarischen Übersicht über das gesamte Epos die Passagen auszuweisen, in denen Lemnius den Acta folgt bzw. wo er Zugaben zu den Acta einfügt. ${ }^{44}$

Lemnius selbst liefert uns ein Zeugnis dafür, dass er sich für die Arbeit an seinem Epos auf historiographische Quellen gestützt hat. In einer autobiographischen Elegie (amor. 4.2), die er an Jakob Travers gerichtet hat, ${ }^{45}$ inszeniert er zunächst den Kampf zwischen Venus und Apollo, die Lemnius zur ihnen jeweils zugehörigen Art der Dichtung ziehen wollen. Apollo fordert ihn auf, die Geschichte seines Landes zu verherrlichen (amor. 4.2.19 patrias graviori carmine laudes), und Lemnius lässt sich überreden (amor. 4.2.55-61 Materiam Phoebo est Oeneia terra datura, / et nostri Martis nobile surget opus [...] Heroes patrios et Martia bella canemus). Es folgt eine umfangreichere Beschreibung, wie lange sich Lemnius mit dem Gedanken trug, ein solches Gedicht zu schreiben, und wie viele Rückschläge es gab. Der Erfolg stellte sich dann endgültig ein, nachdem er in geschichtlichen Aufzeichnungen las und von diesen inspiriert wurde (amor. 4.2.131-134):

Dumque lego patriae nuper mihi praelia gentis, quae secum noster scripta Saletus habet, ipse mihi videorque acies concurrere campis factaque Rhetea bella videre manu.

Während ich nun kürzlich von den Schlachten meines Heimatvolkes las, von denen unser Salet Aufzeichnungen besitzt, schien es mir, als rennten Schlachtreihen im Felde aufeinander los und ich sähe die Kriegstaten, die von rätischer Hand vollbracht worden sind. ${ }^{46}$

Bei dem hier genannten Saletus handelt es sich um Wolfgang Salet, ${ }^{47}$ einen persönlichen Freund, der etwa auch ein Gedicht zu den Paratexten von Lemnius' Odyssee-Übersetzung beigesteuert hat. Er wirkte in Chur als Stadtschreiber und, wie Lemnius auch, als Lehrer in der Nikolai-

44 Vgl. Michel 1914 (wie Anm. 8), 87-104.

45 Edition und Kommentar zu dieser Elegie: Mundt 1988 (wie Anm. 8), 106-115, 160-162; vgl. Plattner 1874 (wie Anm. 5), xxi. Allgemein zu Lemnius'Amores vgl. L. Mundt, "Zu den Amores (1542) des Simon Lemnius", in A. Dalzell, C. Fantazzi, R.J. Schoeck (ed.), Acta Conventus Neo-Latini Torontonensis (New York, 1991), 519-528.

46 Übersetzung: Mundt 1988 (wie Anm. 8), 113.

47 Zu Wolfgang Salet vgl. Merker 1908 (wie Anm. 8), 7; Mundt 1983 (wie Anm. 1), Bd. 1, 254. 
Schule. Für unseren Kontext ist wichtig, dass sich Lemnius als Forscher inszeniert, der anhand von historiographischen Quellen Vorstudien für seine Dichtung betreibt. Mit den Acta konnte exakt die Schrift identifiziert werden, als deren Leser sich Lemnius sich hier in den Amores in Szene setzte. Die Raeteis wurde dann ihrerseits im 16. und 17. Jahrhundert von Historikern intensiv gelesen und fand Eingang in die historiographischen Werke von Ulrich Campell (um 1510-um 1582), Johann Guler von Wyneck $(1562-1637)^{48}$ und Fortunat Sprecher von Bernegg (1585-1647). ${ }^{49}$

\section{Vergleiche mit den Schlachten der Punica}

Eine Technik, die Lemnius anwendet, um in seinen Lesern die enge Verbindung seines Epos mit den Punica zu evozieren, sind Vergleiche seiner Kämpfe mit den großen Schlachten des Zweiten Punischen Krieges. Auch wenn diese Ereignisse rund um Hannibals Einfall in Italien auch in anderen lateinischen Texten zur Sprache kommen, allen voran im Geschichtswerk des Livius, legt die gleich noch en detail zu besprechende Einbettung dieser Vergleiche es nahe, dass Lemnius keine Verbindung $\mathrm{zu}$ irgendeinem Text, der diese Schlachten behandelt, sondern speziell zu den Punica herstellen wollte, wo sie besonders ausführlich geschildert werden. Dadurch werden die einzelnen Gefechte in den Schweizerkriegen gerade mit den Schlachten parallel geführt, die für die künftige Größe Roms entscheidend waren und die ins kollektive Gedächtnis Roms eingegangen sind. Lemnius will etwas Ähnliches für Graubünden schaffen.

Im dritten Buch beschreibt Lemnius, wie Truppen des Schwäbischen Bundes über den Rhein kommen und die Orte Sax, Gams und Grabs zwischen Feldkirch und Vaduz plündern (Raet. 3.379-485). Bei der Abwehr hat sich Ulrich Freiherr von Hohensax (1458?-1538) besonders hervorgetan (Raet. 3.407-409):

48 Die oben erwähnte Handschrift T, auf die sich Plattner bei seiner Ausgabe stützte, ist Teil eines umfangreicheren Codex, der auch historiographische Schriften Gulers enthält.

${ }^{49}$ Zur Rezeption der Raeteis in der späteren Historiographie vgl. Plattner 1874 (wie Anm. 5), xix-xx. 
Ulciscuntur agros hostes poenasque rependunt, praecipue heroi Sacco, qui laudibus aequat

Scipiadas Fabiumque et celsum Marte Neronem.

408 heroi: T Heroi

Für die [Verwüstung der] Felder mussten die Feinde bezahlen und sie wurden bestraft, besonders durch den heldenmütigen Saccus [scil. Freiherr von Hohensax], der im Lob, das ihm zusteht, den Scipiaden, dem Fabius und dem im Krieg erhabenen Nero gleichkommt.

Ulrich wird im letzten hier zitierten Vers mit drei Personen verglichen, die im Zweiten Punischen Krieg auf römischer Seite die entscheidende militärische Rolle spielen: Scipio Africanus wird am Ende der Punica sogar zum dominierenden Charakter, der beenden kann, was sein Vater und sein Onkel (daher der Plural Scipiades) in Spanien begonnen haben; ${ }^{50}$ Fabius, genannt Cunctator, gehörte mit seiner zögerlichen Haltung zu offenen Feldschlachten $\mathrm{zu}$ den letztlich für Rom kriegsentscheidenden Feldherren; ${ }^{51}$ Claudius Nero war für den römischen Sieg am Metaurus verantwortlich. ${ }^{52}$ Lemnius lädt mit diesen drei Vergleichen wohl nicht $\mathrm{zu}$ einem direkten Vergleich zwischen seinem und den römischen Helden ein - der detaillierte Vergleich zwischen Ulrich und Scipio Africanus würde wohl eher schief ausfallen - sondern ruft die Welt der Punica auf, vor deren Hintergrund er sein Gedicht gelesen wissen möchte. Ulrichs Vergleich mit Scipio, Fabius und Claudius Nero mündet dann in eine poetologische Betrachtung: Ulrichs Leistungen für das Vaterland verdienen es,

50 Grundlegend zu Scipio Africanus in den Punica vgl. F. Ahl, M.A. Davis, A.J. Pomeroy, "Silius Italicus", $A N R W$ II 32.4, 2492-2561, hier 2552-2555; Marks 2005 (wie Anm. 35).

51 Zu Fabius' Rolle in den Punica vgl. Ahl, Davis, Pomeroy 1986 (wie Anm. 50), 2523-2531; M. Fucecchi, "The Shield and the Sword. Q. Fabius Maximus and M. Claudius Marcellus as Models of Heroism in Silius' Punica", in A. Augoustakis (ed.), Brill's Companion to Silius Italicus (Leiden - Boston, 2010), 219-239.

$52 \mathrm{Zu}$ Claudius Neros Rolle in den Punica vgl. Ahl, Davis, Pomeroy 1986 (wie Anm. 50), 2540-2542; B. Gibson, "Silius Italicus: A Consular Historian?”, in Augoustakis 2010 (wie Anm. 51), 47-72, hier 49-50.

Humanistica Lovaniensia 70.2 (2021), 185-208 
ewig erinnert zu werden. Dieses ehrende Andenken können nur die $\mathrm{Mu}-$ sen und die (epische) Dichtung gewährleisten. ${ }^{53}$

Das eben beschriebene Beispiel für die Integration der Punica in die Raeteis ist nicht singulär. Während des Kriegsrates, den die Raeter vor der Calven-Schlacht abhalten, ergreift einer ihrer Anführer, Paul von Capol, das Wort und meint, die geplante Schlacht werde besser ausgehen als die bei Cannae (Raet. 6.199-200 Cannis meliori bella geremus / auspicio). Damit stellt er einen direkten Bezug zum 9. Buch der Punica her, in der die für die Römer so katastrophal endende Schlacht bei Cannae geschildert wird.

In der abschließenden Betrachtung zur Calven-Schlacht setzt Lemnius das eben beschriebene Geschehen mit allen großen Schlachten in den Punica gleich (Raet. 7.825-830):

Pugna fuit clara, et toto memorabilis aevo.

Haec Trebiam, haec Cannas, Trasimeni haec littora saeva, et Ticine tuas aequavit sanguine ripas.

Nec minor est Ramus, fuerat quam lympha Metauri;

nec Scamander aqua, Simoëntis et unda cruore

nobilior iactet clades lacrimasque suorum.

826 Cannas: $\mathbf{T}$ camas : $\mathbf{T}^{\mathbf{1}}$ Cannas

829 Simoëntis: $\mathbf{T}$ Simeontis : $\mathbf{T}^{\mathbf{1}}$ Simoëntis

830 lacrimasque: $\mathbf{T}$ lachrymasque

Diese Schlacht war legendär, man wird sich auf ewig an sie erinnern. Sie kam den Schlachten am Trebia, bei Cannae und an den wilden Ufern des Trasimenersees gleich, und auch deinen blutgetränkten Ufern, Ticinus. Der Ram-Bach ist nicht weniger bedeutend als das Wasser des Metaurus. Weder der Skamander noch der blutgetränkte Simoeis kann seine Schlachten und die Tränen seiner Soldaten bedeutender nennen.

Während sich die letzten beiden hier genannten Flüsse, die Brüder Skamander und Simoeis, auf die Belagerung Trojas und somit auf Homers

53 Raet. 3.410-418 Qui bello patrio hoc virtutum fortibus ausis / egregiam laudem retulit, palmamque reportans / ullo facta dedit nunquam reticenda sub aevo, / quae dudum aeternam laudem meruere Camenis, / quorum perpetuis volitabit gloria pennis, / heroësque equitesque inter splendebit honore, / si quid Pieridum Musarum carmina possunt. / Sacce, decus Martis, Raeteae et gloria gentis, / summe nepos Sacci, deducte ab origine Tusca. 
Ilias beziehen, ${ }^{54}$ führen alle weiteren fünf Vergleiche zu den Punica, und zwar zu den Schlachten am Trebia (Pun. 3.480-703), bei Cannae (Pun. 8.242-10,336), am Trasimenersee (Pun. 3.704-6,53), am Ticinus (Pun. 3.81-479) und am Metaurus (Pun. 15.626-823). - Ganz ähnlich sind die Vergleiche, die Lemnius im 8. Buch der Raeteis für die Kämpfe zwischen den Truppen Maximilians und den Eidgenossen wählt (Raet. 8.552-557):

[...] Haud Troiani proelia belli,

nec sontes Thebae poterunt aequare furorem, nec toties Ticine rubens fatalibus armis, nec Trebia aut Trasimene lacus, sed sanguis inundans ad Latias Cannas, at non superare furore, insanisque animis, rabieque furente per arma.

Nicht die Schlachten des Trojanischen Krieges, nicht das schuldbeladene Theben wird diesem Rasen gleichkommen können, nicht du, Ticinus, der du so oft von todbringenden Waffen rot gefärbt wurdest, nicht der Trebia oder der Trasimenersee, sondern das mit Blut getränkte latinische Cannae, doch selbst das kann diese Raserei nicht übertreffen, die kranke Wut und den in Waffen rasenden Zorn.

Mit dem Trojanischen Krieg ist hier wieder auf Homer und seine Ilias angespielt, mit sontes Thebae auf Statius und seine Thebais, doch die restlichen vier Beispiele führen wieder zu Silius Italicus. Solche Vergleiche dienen der Leserlenkung und sie rufen gerade nicht nur die Ereignisse des Zweiten Punischen Krieges auf, sondern durch die Verbindung mit anderen großen epischen Schlachten eben das Epos des Silius Italicus. Somit werden auch die Leser, denen die intertextuellen Verweise entgangen sind, expressis verbis auf den Vergleich mit Silius Italicus gestoßen.

\section{Schlussfolgerungen}

Es wurde wiederholt festgestellt, dass sich in der Mitte des 16. Jahrhunderts Vergils Aeneis als das zentrale Vorbild für neulateinische Epik durchgesetzt hatte. ${ }^{55}$ Auch Lemnius wollte das Prestige, das mit einem

54 Skamander z.B. Hom. Il. 21.213, Simoeis z.B. Hom. Il. 4.475. Homer spielt im literarischen Schaffen des Simon Lemnius eine besondere Rolle, hat er doch eine 1539 eine Elegia in commendationem Homeri de bello Troiano (Edition bei Mundt 1988a (wie Anm. 8), 205-222) und 1549 in Basel eine lateinische Übersetzung der Odyssee und der Batrachomyomachia veröffentlicht.

55 Vgl. Schaffenrath 2015 (wie Anm. 28). 
Gedicht im Stile Vergils verbunden war, für sich nutzen. Bereits in der oben besprochenen Elegie an Jakob Travers sieht er sich als Vergil seiner Heimat (amor. 4.2.66 Et tibi Virgilium patria rura dabunt). Lemnius weiß, dass er durch Vergilnachfolge nicht nur seinen eigenen Ruhm als Dichter befördern kann; er kann dadurch auch ewigen Ruhm für die von ihm geschilderten Personen begründen. So etwa spricht er im vierten Buch mit Benedikt Fontana einen seiner wichtigsten Helden direkt an (Raet. 4.906-908):

Si qua fidem nostra est operi latura propago

Raetorum, non te iuvenis memorande silebo, at qui te heroum Fontanee maxime dicam.

Wenn das Volk der Raeter unserem Werk nur Glauben schenken will, werde ich dich nicht mit Schweigen übergehen, denkwürdiger junger Mann, sondern ich werde dich den größten meiner Helden nennen, Fontana!

Wie sehr Lemnius mit Ankündigungen wie dieser Recht behalten sollte, zeigt die spätere Rezeption des Benedikt Fontana, der es nach seiner würdigenden Darstellung in der Raeteis zum Graubündner Nationalhelden gebracht hat. Die Statue, die ihm zu Beginn des 20. Jahrhunderts in Chur in dem nach ihm benannten Park errichtet wurde (s. Abb. 1), ist nur eines von vielen Beispielen, wie man Fontana in der Kunst für nationale Identitätsbildung einsetzen konnte, während sich die historische Kritik v.a. des 19. Jahrhunderts klarerweise schwer mit ihm tat. ${ }^{56}$

Lemnius konnte viel von Silius Italicus lernen, denn auch er stellte sich in die Nachfolge Vergils und sah sein Ziel darin, ein ganzes Volk in seinem Fall, die Römer - zu rühmen. Lemnius kopiert seine Technik und schafft so, nach dem Vorbild der Punica, ein Nationalepos für sein Volk, die Raeter.

56 Vgl. C. Jecklin, "Benedict Fontana und die historische Kritik", in Beilage zum Programm der Kantonsschule (Chur, 1886); Willi 1971 (wie Anm. 6).

${ }_{57}$ Ein Beispiel für den Durchgriff auf die Aeneis stehe hier stellvertretend für viele: Die Eidgenossen, die die Truppen des Schwäbischen Bundes zurückschlagen, werden im vierten Buch (Raet. 4.766-773) gleich wie die Römer in der Römerschau der Aeneis (Aen. 6.845-847) dargestellt: mild $\mathrm{zu}$ den Besiegten, hart $\mathrm{zu}$ denen, die sich ihnen widersetzen. 
In der ersten Hälfte des 16. Jahrhunderts war Silius Italicus ein recht beliebter Autor: ${ }^{57}$ Seit der editio princeps, die 1471 in Rom erschien (Hain 14733), verzeichnet Michael von Albrecht allein bis ins Jahr 1550 nicht weniger als 20 Ausgaben. ${ }^{58}$ Es ist nicht unwahrscheinlich, dass Lemnius die Punica in der Ausgabe gekannt hat, die 1543 in Basel bei Henricpetri (per Henricum Petrum) erschienen ist. Dabei handelt es sich um eine schlichte Textausgabe, in der der Text der Punica um eine Biographie des Silius Italicus und um kurze metrische Zusammenfassungen aller 17 Bücher erweitert wurde. Das Epos selbst ist dann in Paragraphen eingeteilt, denen jeweils eine kurze Zusammenfassung in Prosa vorausgeht.

Der relativ hohe Bekanntheitsgrad der Punica und das Vorbild, das sie für eine mögliche Benutzung der Aeneis darstellten, legten es für Simon Lemnius nahe, sich ihrer zu bedienen und sein Epos über das rätische Volk ganz nach dieser Vorlage zu gestalten. Er feiert sein Volk, für das er eine verbindliche Heldenerzählung kreiert, und sichert sich dadurch gleichzeitig seinen Ruhm als Dichter seiner Heimat.

Ludwig Boltzmann Institute for Neo-Latin Studies florian.schaffenrath@neolatin.lbg.ac.at

57 Zur Rezeptionsgeschichte des Silius Italicus im 16. Jahrhundert vgl. F. Schaffenrath, "Silius Italicus (Tiberius Catius Asconius Silius Italicus). Punica", in C. Walde (ed.), Die Rezeption der antiken Literatur. Kulturhistorisches Werklexikon (Stuttgart - Weimar, 2010), 911-922.

58 M. von Albrecht, Silius Italicus. Freiheit und Gebundenheit römischer Epik (Amsterdam, 1964), 215-216.

Humanistica Lovaniensia 70.2 (2021), 185-208 


\section{Abbildungen}

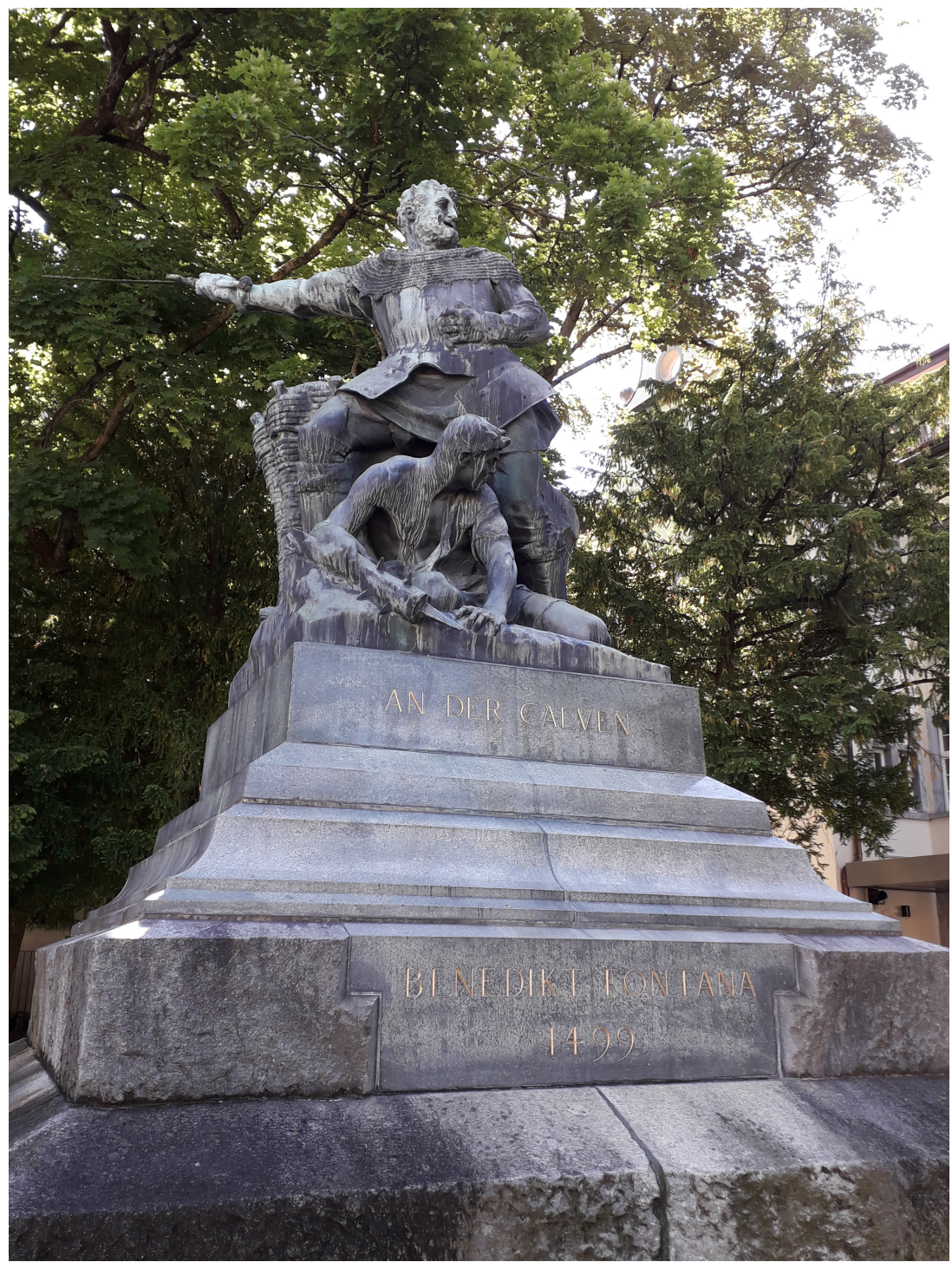

Abb. 1: Bildnis des Benedikt Fontana von Richard Kissling (1848-1919) im Fontanapark in Chur (Bild: F. Schaffenrath). 